05,12

\title{
Особенности релаксации остаточной намагниченности антиферромагнитных наночастиц на примере ферригидрита
}

\author{
(ㄷ Д.А. Балаев ${ }^{1,2}$, А.А. Красиков ${ }^{1}$, А.Д. Балаев ${ }^{1}$, С.В. Столяр ${ }^{1,2,3}$, В.П. Ладыгина ${ }^{2}$, Р.С. Исхаков ${ }^{1}$ \\ ${ }^{1}$ Институт фризики им. Л.В. Киренского ФИЦ КНЦ СО РАН, \\ Красноярск, Россия \\ ${ }^{2}$ Сибирский фредеральный университет, \\ Красноярск, Россия \\ ${ }^{3}$ ФИЦ КНЦ СО РАН, \\ Красноярск, Россия \\ E-mail: dabalaev@iph.krasn.ru
}

Поступила в Редакцию 20 февраля 2020 г.

В окончательной редакции 20 февраля 2020 г.

Принята к публикации 20 февраля 2020 г.

\begin{abstract}
Проведено исследование релаксации остаточной намагниченности антиферромагнитно упорядоченных наночастиц ферригидрита в условиях реализации для этих систем эффекта обменного смещения. Релаксация намагниченности характеризуется логарифмической зависимостью от времени, типичной для термоактивационных процессов перескока магнитного момента частиц через потенциальные барьеры, вызванные магнитной анизотропией. Полученная из обработки данных релаксации остаточной намагниченности величина энергии барьеров (в условиях охлаждения в поле) значительно превосходит таковую для обычных условий (охлаждение без поля). Обнаруженное различие указывает на возможность использования процесса релаксации остаточной намагниченности для анализа механизмов, приводящих к эффекту обменного смещения в антиферромагнитных наночастицах и измерения параметров, характеризующих обменную связь магнитных подсистем в таких объектах.
\end{abstract}

Ключевые слова: антиферромагнитные наночастицы, ферригидрит, обменное смещение, релаксация намагниченности.

DOI: $10.21883 /$ FTT.2020.07.49469.038

\section{1. Введение}

Магнитные свойства наночастиц антиферромагнитных (АФМ) материалов качественно отличаются от магнитных свойств массивных антиферромагнетиков. Это вызвано определяющей ролью поверхностных эффектов при уменьшении размеров частиц. Однако и объемные дефекты наночастиц антиферромагнетика также важны: их наличие приводит к декомпенсации спинов в подрешетках и появлению у АФМ-наночастиц нескомпенсированного магнитного момента [1-3]. И, как следствие, в АФМ-наночастицах формируется несколько магнитных подсистем: ферромагнитная (ФМ) подсистема (нескомпенсированный момент) [4-15], поверхностные спины [9,14-24], антиферромагнитно упорядоченное ,ядро“ [25]. Наличие указанных подсистем и их взаимодействие приводит к ряду ярких эффектов, наблюдаемых при изучении магнитных свойств АФМ-наночастиц [18-22]. Одним из таких эффектов является смещение петли магнитного гистерезиса после охлаждения во внешнем поле.

Данный эффект, называемый обменным смещением, был впервые обнаружен на субмикронных частицах $\mathrm{Co} / \mathrm{CoO}[26]$ с выраженной магнитной структурой $\Phi \mathrm{M}(\mathrm{Co}) / \mathrm{A} \Phi \mathrm{M}(\mathrm{CoO})$. В случае субмикронных частиц, либо в идеализированном варианте - тонкопленочные структуры типа $Ф \mathrm{M} / \mathrm{AФМ} \mathrm{-} \mathrm{смещение} \mathrm{петли} \mathrm{маг-}$ нитного гистерезиса по оси абсцисс (внешнее поле) имеет место после охлаждения системы во внешнем магнитном поле от температуры, превышающей точку Нееля антиферромагнетика (либо точку Кюри ферромагнетика) [27-29]. Обменная связь ферромагнетика и антиферромагнетика на поверхности раздела $\Phi \mathrm{M} / \mathrm{A} Ф$ является причиной наблюдаемого эффекта [27-29].

Однако достаточно часто аналогичный эффект наблюдается и на химически однородных наночастицах АФМ-материалов $[11,12,20,30-43]$. И здесь необходимым условием для реализации эффекта обменного смещения является наличие, как минимум, двух магнитных подсистем. Для экспериментального наблюдения эффекта обменного смещения в АФМ-наночастицах достаточно проводить охлаждение во внешнем поле от температуры, превышающей температуру суперпарамагнитной (СПМ) блокировки, которая, как правило, ниже температуры Нееля. Кроме того, для АФМ-наночастиц зачастую наблюдается и смещение петли магнитного гистерезиса по оси ординат. Отметим, что механизмы, приводящие к эффекту обменного смещения в АФМ-наночастицах, пока не ясны, также не выделены магнитные характеристики, измерение которых даст достоверную информацию для определения величины обменной связи магнитных подсистем. 
Ферригидрит (номинальная формула $\mathrm{Fe}_{2} \mathrm{O}_{3} \cdot n \mathrm{H}_{2} \mathrm{O}$ ) обладает АФМ-упорядочением и существует только в виде наночастиц размерами не более $8 \mathrm{~nm}$. Он входит в состав ферритина, играющего важную роль в жизнедеятельности живых организмов. Ферригидрит может быть получен химическими методами [44], либо выделен из результатов жизнедеятельности микроорганизмов $[45,46]$. В ряде работ на образцах ферригидрита наблюдалось смещение петли магнитного гистерезиса после охлаждения от температуры, превышающей температуру СПМ-блокировки [11,12,36-43]. Однако обычно наночастицы ферригидрита демонстрируют достаточно высокие поля (порядка $\left.10^{5} \mathrm{Oe}\right)$ необратимого поведения намагниченности, и существует проблема разделения эффекта обменного смещения от эффекта, связанного с частной петлей гистерезиса [40-42]. В работе [42] был предложен метод, позволяющий выявить эффект обменного смещения и установить, что для исследованной серии образцов ферригидрита этот эффект наблюдается при размере наночастиц более трех нанометров.

Гистерезис любой физической величины связан с релаксационными процессами, и логично, что эффект обменного смещения должен также отражаться в релаксации намагниченности. В данной работе исследована релаксация остаточной намагниченности образца ферригидрита (средний размер частиц $4 \mathrm{~nm}$ ) в условиях реализации обменного смещения (охлаждения во внешнем поле) и в обычных условиях (охлаждения в нулевом поле). Основной целью работы было выявление возможности использования данных по релаксации намагниченности для установления параметров, характеризующих обменную связь магнитных подсистем в исследуемых наночастицах антиферромагнитно упорядоченного ферригидрита.

\section{2. Эксперимент}

Образцы ферригидрита биогенного происхождения выделялись из бактериальных осадков после культивирования бактерий Klebsiella oxytoca в анаэробных условиях, как описано в работах $[11,45,46]$. Получаемый высушенный золь представляет собой агрегированную систему покрытых органической оболочкой [47] наночастиц ферригидрита с малым средним размером $\sim 2-3 \mathrm{~nm}$. Получаемые таким образом системы различных партий демонстрируют идентичные размеры частиц, а характерные температуры СПМ-блокировки ТВ находятся в узком интервале $(23-25 \mathrm{~K}$ в поле до $1 \mathrm{kOe})[11,13,43]$. Ранее было показано, что низкотемпературный отжиг (до $\sim 200^{\circ} \mathrm{C}$ ) ферригидрита в воздушной атмосфере приводит к контролируемому увеличению размеров частиц в агрегированных системах $[12,13,48]$, тогда как образование других модификаций оксида или гидроксида железа при таком отжиге не происходит. Образец, на котором в данной работе исследовалась релаксация намагниченности и петли магнитного гистерезиса, прошел отжиг при $200^{\circ} \mathrm{C}$ в течение $24 \mathrm{~h}$. Средний размер частиц этой системы (по данным просвечивающей электронной микроскопии) составил $\sim 4 \mathrm{~nm}$ [42]. Далее этот агрегат обозначен как $\mathrm{FH}-4 \mathrm{~nm}$.

Магнитные измерения проводились на вибрационном магнетометре [49]. Исследуемый порошок фиксировался в измерительной капсуле в парафине. Для измерений температурных зависимостей намагниченности $M(T)$ использовались режимы охлаждения без поля (ZFC zero field cooling) и во внешнем поле (FC - field cooling). Петли гистерезиса намагничивания $M(H)$ в режиме ZFC при $T=4.2 \mathrm{~K}$ были измерены до различных значений максимального приложенного поля $\pm H_{\max }$ с последовательно увеличивающейся величиной $\left|H_{\max }\right|$. Зависимости $M(H)$ в режиме FC (при $T=4.2 \mathrm{~K}$ ) были измерены при охлаждении в поле $H_{\mathrm{FC}}=+30 \mathrm{kOe}$, $+45 \mathrm{kOe}$ от температуры $120 \mathrm{~K}$; эта температура значительно превышает температуру необратимого поведения зависимостей $M(T)$.

Релаксация намагниченности измерялась при $T=4.2 \mathrm{~K}$ после выполнения условий $\mathrm{FC}$ при $H_{\mathrm{FC}}=+30 \mathrm{kOe} \quad$ и последующего уменьшения поля до нулевого значения, а также в условиях ZFC после приложения поля до величины $30 \mathrm{kOe}$ и уменьшении поля до нуля. В обоих случаях фиксировалось уменьшение остаточной намагниченности $M_{R}$ со временем зависимость $M_{R}(t)$. Для измерений этого типа, после „остановки“ при $H=0$ в течение $4500 \mathrm{~s}$, измерение петли гистерезиса продолжалось.

\section{3. Результаты и обсуждение}

На рис. 1 приведены зависимости $M(T)$ исследованного образца наночастиц ферригидрита в режимах ZFC и FC в различных магнитных полях. Из данных рис. 1 видно типичное поведение для СПМ-систем: немонотонное поведение зависимостей $M(T)_{\mathrm{ZFC}}-$ наличие максимума при характерной температуре СПМ-блокировки (при $H=1 \mathrm{kOe}, T_{B} \approx 60 \mathrm{~K}$ ), расхождение зависимостей $M(T)_{\mathrm{ZFC}}$ и $M(T)_{\mathrm{FC}}$ в окрестности температуры $T_{B}$ и сдвиг $T_{B}$ в область низких температур при увеличении внешнего поля.

На рис. 2 представлены петли магнитного гистерезиса $M(H)$ при $T=4.2 \mathrm{~K}$, полученные в режиме ZFC при значениях максимального приложенного поля $H_{\max }$, равных \pm 30 и $\pm 60 \mathrm{kOe}$, а также $M(H)$ в режиме FC при $H_{\mathrm{FC}}=+30 \mathrm{kOe}$. Виден значительный сдвиг петли гистерезиса в режиме FC, как по оси абсцисс, так и по оси ординат. На рис. 3 показаны участки зависимостей $M(H)$ для условий $\mathrm{FC}$ и $\mathrm{ZFC}$ в окрестности пересечения с осью ординат в положительной области значений $M$. Здесь приведены данные для двух видов измерений: 1) стандартная запись петли в диапазоне $\pm H_{\mathrm{FC}}$, либо $\pm H_{\max }$ в случае ZFC, 2) запись петли с „остановкой“ при $H=0$ в течение $4500 \mathrm{~s}$. Заметная релаксация остаточной 


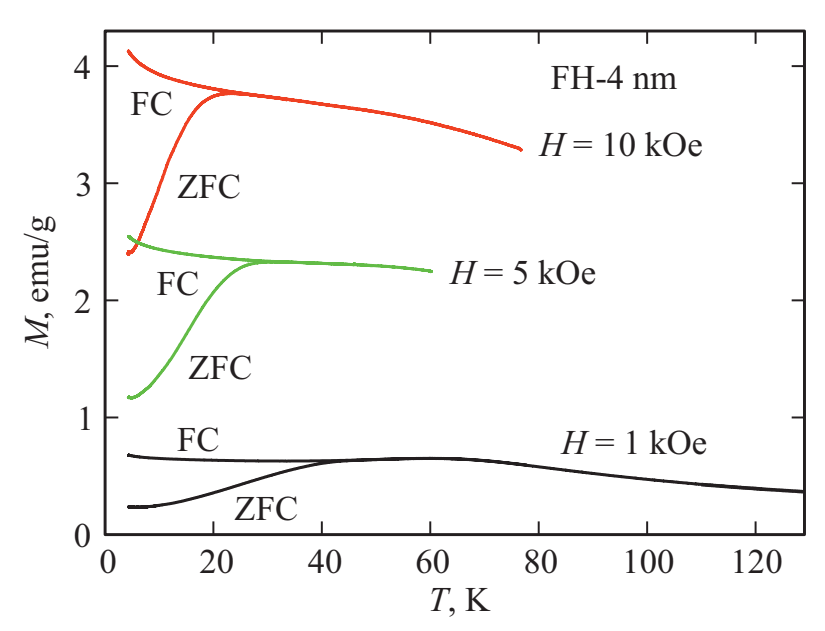

Рис. 1. Зависимости $M(T)$ исследованного образца FH-4 nm в режимах ZFC и $\mathrm{FC}$ в различных магнитных полях.

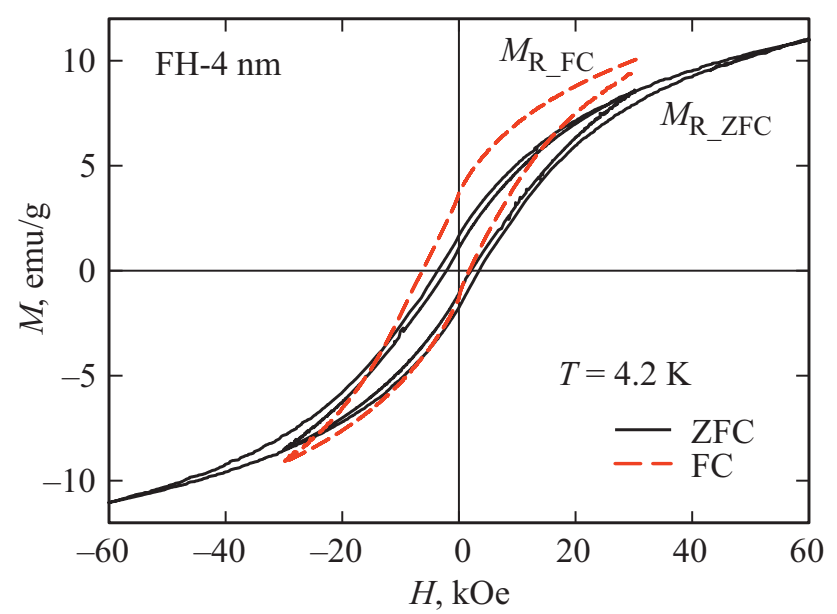

Рис. 2. Зависимости $M(H)$ (при $T=4.2 \mathrm{~K}$ ) в условиях ZFC (при $H_{\max }=30$ и $60 \mathrm{kOe}$ ) и $\mathrm{FC}$ при $H_{\mathrm{FC}}=+30 \mathrm{kOe}$.

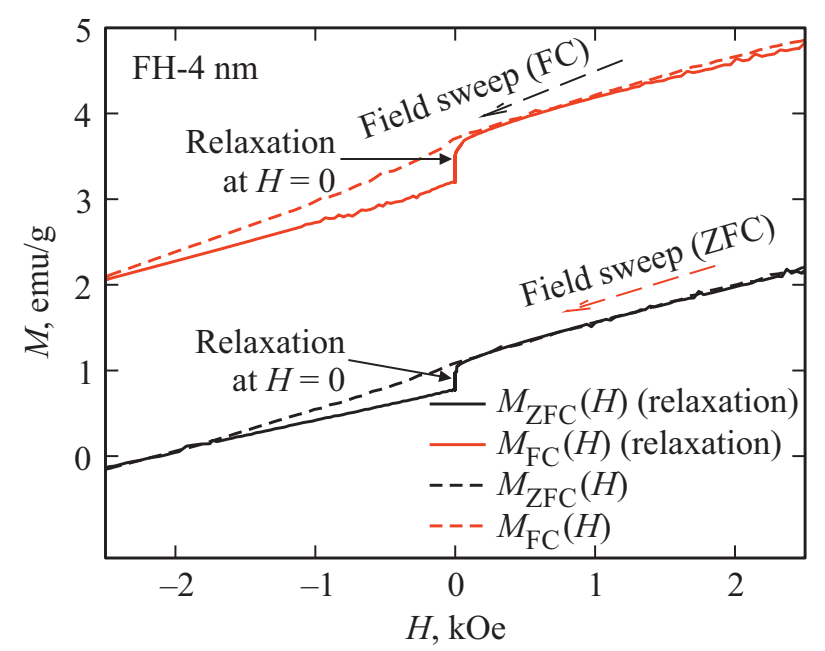

Рис. 3. Участки зависимостей $M(H)$ в условиях $\mathrm{FC}$ (при $H_{\mathrm{FC}}=+30 \mathrm{kOe}$ ) и ZFC (при $H_{\max }=30 \mathrm{kOe}$ ), полученные как стандартным образом, так и с остановкой при $H=0$ с записью релаксации остаточной намагниченности $M_{\mathrm{R} \_ \text {FC }}$ в течение $4500 \mathrm{~s}$. намагниченности $M_{\mathrm{R} \_ \text {FC }}$ (режим FC) и $M_{\mathrm{R} \_Z F C}$ (режим $\mathrm{ZFC}$ ) наблюдается для обеих магнитных предысторий.

Обычно параметром, характеризующим обменное смещение в магнитных ФМ/АФМ-структурах, является величина

$$
H_{\mathrm{EB}}=-\left(H_{\mathrm{CL}}+H_{\mathrm{CR}}\right) / 2,
$$

где $H_{\mathrm{CL}}$ и $H_{\mathrm{CR}}-$ соответственно „левая“ и „правая“ коэрцитивная сила петли гистерезиса в условиях FC при $H_{\mathrm{FC}}>0$ [27-29]. Очевидно, что $H_{\mathrm{EB}} \neq 0$ при $\left|H_{\mathrm{CL}}\right|>\left|H_{\mathrm{CR}}\right|$, или, что эквивалентно, при $\left|H_{\mathrm{CL}}\right|>\left|H_{\mathrm{C}}\right|$ (здесь и далее $H_{\mathrm{C}}$ - коэрцитивная сила на симметричной петле, полученной в условиях ZFC). Однако петли гистерезиса исследованного образца $\mathrm{FH}-4 \mathrm{~nm}$ остаются открытыми в условиях ZFC в полях вплоть до $60 \mathrm{kOe}$ (см. рис. 2), и значение $H_{\mathrm{C}}$ полной петли гистерезиса неизвестно. Тогда выражение (1) становится неприменимым, поскольку сдвиг петли может быть просто следствием частной петли гистерезиса [29]. Следовательно, необходимо определить величину коэрцитивной силы для условий ZFC при „большом“ значении $H_{\max }$ путем экстраполяции зависимости $H_{\mathrm{C}}\left(H_{\max }\right)$ к некоторому постоянному значению $H_{\mathrm{C} \_ \text {INF }}$ при $H_{\max } \rightarrow \infty$. Тогда можно сравнивать значения $\left|H_{\mathrm{CL}}\right|$ и $H_{\mathrm{C} \_\mathrm{INF}}$, а поле обменного смещения определять уже по формуле [42]

$$
H_{\mathrm{EB}}=\left|H_{\mathrm{CL}}\right|-\left|H_{\mathrm{C}_{-} \mathrm{INF}}\right| \text {. }
$$

В принципе, зависимость $H_{\mathrm{C}}\left(H_{\max }\right)$ может быть аппроксимирована некоторой функцией, описывающей экспериментальные точки $H_{\mathrm{C}}, H_{\max }$ и стремящейся к насыщению при больших значениях $H_{\max }$. В работе [41] было показано, что применительно к АФМ-наночастицам справедливо выражение

$$
H_{\mathrm{C}}\left(H_{\max }\right)=H_{\mathrm{C} \_\mathrm{INF}}\left[1-H^{*} / H_{\max }\right]^{b}
$$

c показателем степени $b=1.5$ для ферригидрита. Обработка экспериментальных данных, полученных на исследованном образце $\mathrm{FH}-4 \mathrm{~nm}$, дает значение $\left|H_{\mathrm{C} \_\mathrm{INF}}\right| \approx 4.75 \mathrm{kOe}$, что соответствует $H_{\mathrm{EB}} \approx 1.25 \mathrm{kOe}$ при $\left|H_{\mathrm{CL}}\right| \approx 6 \mathrm{kOe}$.

Рассмотрим сдвиг петли магнитного гистерезиса по оси ординат для условий FC. Величина остаточной намагниченности после охлаждения во внешнем поле $M_{\mathrm{R} \_F C}$ значительно превосходит остаточную намагниченность в условиях ZFC $-M_{\mathrm{R} \_Z F C}$ (см. рис. 2,3). Однако $M_{\mathrm{R} \_Z F C}$, как и $H_{\mathrm{C}}$, зависит от величины $H_{\max }$. Выше была описана процедура определения величины

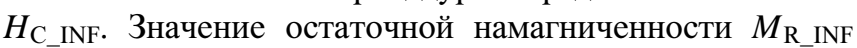
для условий ZFC при „большом“ значении $H_{\max }$ также можно определить путем экстраполяции зависимости $M_{\mathrm{R} \_Z F C}$ от $H_{\max }$ к некоторому постоянному значению при $H_{\max } \rightarrow \infty$. Данные, представленные на рис. 4, иллюстрируют искомую зависимость $M_{\mathrm{R} \_Z F C}$ от $H_{\max }$, полученную из частных петель гистерезиса в условиях ZFC. Видно, что эта зависимость также имеет тенденцию выхода на насыщение (плато) при больших значениях 


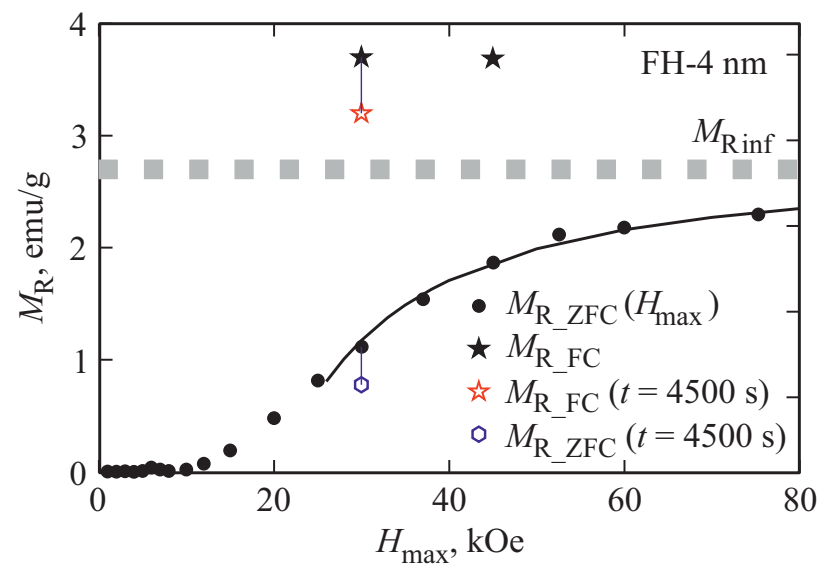

Pис. 4. Значения остаточной намагниченности $M_{\mathrm{R} \_ \text {ZFC }}$ от максимального приложенного поля $H_{\max }$ в условиях ZFC. Также показаны величины остаточной намагниченности в условиях $\mathrm{FC}-M_{\mathrm{R} \_ \text {FC }}$ в полях 30 и $45 \mathrm{kOe}$ (положение точек

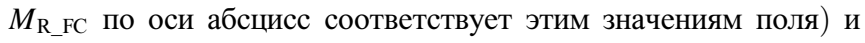
величины остаточной намагниченности после релаксации в течение $4500 \mathrm{~s}-M_{\mathrm{R} \_ \text {FC }}(t=4500 \mathrm{~s})$ и $M_{\mathrm{R} \_ \text {ZFC }}(t=4500 \mathrm{~s}$ ) (соединены вертикальными линиями со значениями $M_{\mathrm{R} \_\mathrm{FC}}(t=0)$ и $\left.M_{\mathrm{R} \_z F C}(t=0)\right)$. Сплошная кривая построена по выражению (4) при $H^{*}=22 \mathrm{kOe}, b=1.5$ и $M_{\mathrm{R} \_\mathrm{INF}}=2.7 \mathrm{emu} / \mathrm{g}$. Положение $M_{\mathrm{R} \_\mathrm{INF}}$ относительно других данных показано горизонтальной штриховой линией.

$H_{\max }$. Анализ данных рис. 4 показал, что зависимость $M_{\mathrm{R} \_ \text {ZFC }}\left(H_{\max }\right)$ в области достаточно больших полей может быть описана функцией, подобной (3):

$$
M_{\mathrm{R} \_\mathrm{ZFC}}\left(H_{\max }\right)=M_{\mathrm{R} \_\mathrm{INF}}\left[1-H^{*} / H_{\max }\right]^{b}
$$

с показателем степени $b=1.5 \pm 0.2$, изменение $b$ в указанных пределах слабо влияет на величину $M_{\mathrm{R} \_\mathrm{INF}}$. Сплошная кривая на рис. 4 построена по выражению (4) при $b=1.5$ и соответствует наилучшей подгонке экспериментальных точек. Горизонтальная пунктирная линия соответствуют величине $M_{\text {R_INF }}$ в выражении (4), а толщина линий демонстрирует оцененную погрешность определения $M_{\mathrm{R} \_I N F}$. На рис. 4 также показаны величины остаточной намагниченности петель $M(H)$ в условиях $\mathrm{FC}-M_{\mathrm{R} \_\mathrm{FC}}$ при $H_{\mathrm{FC}}=30$ и $45 \mathrm{kOe}$ (положение точек $M_{\mathrm{R} \_\mathrm{FC}}$ по оси абсцисс соответствует значению поля $H_{\mathrm{FC}}$, при котором охлаждался образец). Видно, что величина поля, в котором охлаждался образец, практически не влияет на величину остаточной намагниченности $\left(M_{\mathrm{R} \_\mathrm{FC}}\left(H_{\mathrm{FC}}=30 \mathrm{kOe}\right) \approx M_{\mathrm{R} \_ \text {FC }}\left(H_{\mathrm{FC}}=45 \mathrm{kOe}\right)\right)$. Кроме того, также видно, что значение $M_{\mathrm{R} \_\mathrm{FC}}$ значительно пре-

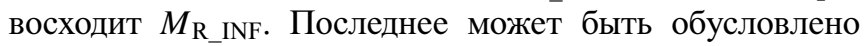
эффектом обменного смещения.

Для описания эффекта обменного смещения в магнитных ФМ/АФМ-пленочных структурах вводится характеристика - удельная энергия связи $\sigma_{\mathrm{S}}$ на поверхности раздела ФМ/АФМ. Для таких „идеальных“, с точки зрения морфологических параметров, объектов величину $\sigma_{\mathrm{S}}$ можно получить, зная намагниченность насыщения ферромагнетика $M_{\mathrm{S}}$, толщину его слоя $d_{\mathrm{FM}}$ и поле обменного смещения $H_{\mathrm{EB}}$ [27-29,50]

$$
\sigma_{\mathrm{S}}=H_{\mathrm{EB}} \cdot M_{\mathrm{S}} \cdot d_{\mathrm{FM}} .
$$

В случае „морфологически не идеальных“ объектов - АФМ-наночастиц, анализ в рамках выражения (5) возможен только при некоторых допущениях. Во-первых, основной вклад в гистерезис $M(H)$ дают нескомпенсированные магнитные моменты частиц (ФМ-подсистема), тогда обменная связь реализуется как между ФМ-подсистемой и АФМ-упорядоченным „ядром“ частицы, так и между ФМ-подсистемой и подсистемой поверхностных спинов. Во-вторых, параметр $d_{\mathrm{FM}}-$ толщина слоя ФМ-подсистемы в нашем случае неопределен, однозначно можно указать только неравенство $d_{\mathrm{FM}} \leq D(D-$ размер частицы $)$. И, наконец, необходимы корректные определения значений $H_{\mathrm{EB}}$ (см. выше) и намагниченности насыщения ФМ-подсистемы (здесь необходимы большие внешние поля для достижения насыщения вклада от ФМ-подсистемы, что проявляется как выход зависимости $M(H)$ на линейный по полю участок $[8,10,14,15,25])$. Оценки удельной энергии связи $\sigma_{\mathrm{S}}$ по выражению (5) для АФМ-наночастиц $\mathrm{CuO}$ и $\mathrm{NiO}$ проводились в работах $[31,32]$, причем в качестве $d_{\mathrm{FM}}$ использовался размер частиц $D$.

Обменная связь магнитных подсистем должна проявляться и в величине остаточной намагниченности в условиях FC. По аналогии с выражением (2) можно записать для „избыточной“ остаточной намагниченности $M_{\mathrm{R} \_ \text {вв }}$ следующее выражение:

$$
M_{\mathrm{R} \_\mathrm{EB}}=M_{\mathrm{R} \_\mathrm{FC}}-M_{\mathrm{R} \_\mathrm{INF}} .
$$

Величина $M_{\mathrm{R} \_ \text {Ев }}$ может зависеть от энергии связи $\sigma_{\mathrm{S}}$ достаточно сложным образом, пэтому напрямую делать оценки $\sigma_{\mathrm{S}}$ из $M_{\mathrm{R} \_ \text {вB }}$ проблематично. Тем не менее, обменная связь может проявляться в релаксации остаточной намагниченности - зависимости $M_{\mathrm{R} \_\mathrm{FC}}(t)$. На рис. 4 показаны значения $M_{\mathrm{R} \_F C}$ и $M_{\mathrm{R} \_\mathrm{ZFC}}$ после релаксации в течение $4500 \mathrm{~s}$. Видно, что величина $M_{\mathrm{R} \_\mathrm{FC}}(t=4500 \mathrm{~s})$ значительно превышает $M_{\mathrm{R} \_\mathrm{INF}}$, и, видимо, такое поведение характерно для систем, в которых реализуется эффект обменного смещения.

Релаксация остаточной намагниченности однодоменных магнитных частиц связана с изменением проекций их магнитных моментов относительно направления приложенного поля. При этом магнитные моменты должны преодолевать потенциальные барьеры, вызванные магнитной анизотропией, благодаря тепловым флуктуациям. Рассмотрение вероятности перескоков магнитных моментов частицы через потенциальные барьеры высотой $U[51]$ приводит к часто наблюдаемой логарифмической зависимости намагниченности от времени

$$
M(t)=M\left(t_{0}\right)\left(1-\left(k_{B} T / U\right) \ln \left(t / t^{*}\right)\right) .
$$

В этом выражении $M\left(t_{0}\right)$ - намагниченность в начальный момент времени, $k_{B}$ - константа Больцмана, 


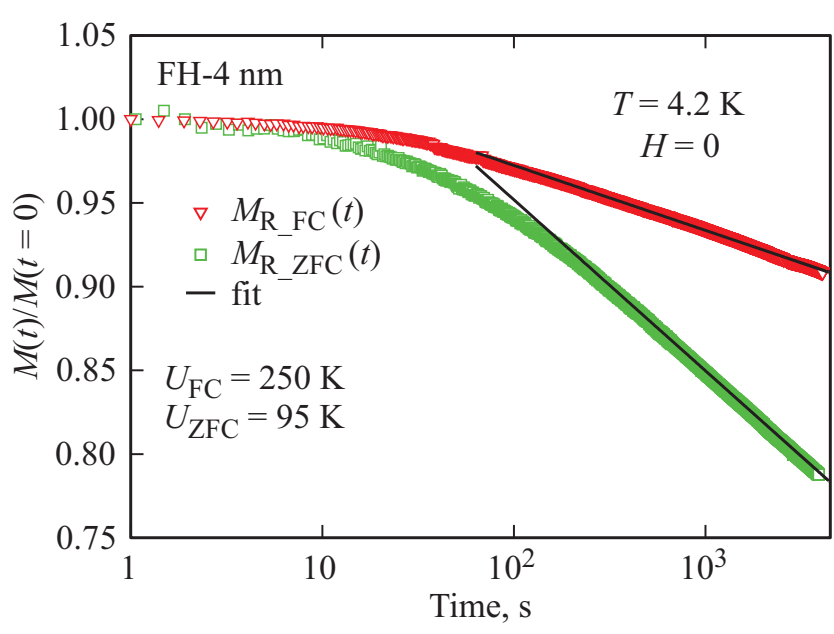

Рис. 5. Нормированные зависимости остаточной намагниченности от времени (логарифмическая шкала) в условиях $\mathrm{FC}$ : $M_{\mathrm{R} \_F C}(t) / M_{\mathrm{R} \_ \text {FC }}(t=0)$ и ZFC: $M_{\mathrm{R} \_ \text {ZFC }}(t) / M_{\mathrm{R} \_ \text {ZFC }}(t=0)-$ символы. Сплошные линии построены по выражению (7) из условий наилучшего согласия с экспериментальными данными при $t>10^{2} \mathrm{~s}$.

$t^{*}$ - константа. Логарифмическая зависимость вида (7) обычно наблюдается при $t \geq 10^{2} \mathrm{~s}$ [51-53]. Величина потенциального барьера $U$ для условия $H=0$ (после „выключения“ поля) должна определяться величиной барьеров, возникших после приложения поля. Следовательно, величина $U$, определяемая из релаксационных измерений в условиях FC при $H=0$, должна содержать вклад от энергии обменной связи магнитных подсистем в наночастице.

На рис. 5 приведены полученные для исследованного образца нормированные зависимости остаточной намагниченности $M_{\mathrm{R} \_\mathrm{FC}}(t) / M_{\mathrm{R} \_\mathrm{FC}}(t=0)$ и $M_{\mathrm{R} \_Z F C}(t) / M_{\mathrm{R} \_Z F C}(t=0)$. На этом рисунке по оси абсцисс использована логарифмическая шкала. Для полученных данных логарифмическая зависимость (7) выполняется, начиная от $t^{*} \sim 10^{2} \mathrm{~s}$. Наклоны релаксационных зависимостей заметно различаются для случаев FC и ZFC. Для зависимости $M_{\mathrm{R} \_\mathrm{FC}}(t)$ величина $U$, определяемая по выражению (7), составляет $250 \mathrm{~K}$, в то время как для условий ZFC энергия барьера $U$ равна $95 \mathrm{~K}$. Такая разница свидетельствует о значительном увеличении высоты энергетических барьеров для магнитных моментов в условиях $\mathrm{FC}$, и очевидно, отражает обменную связь магнитных подсистем в наночастицах.

Проведем оценки удельной энергии связи магнитных подсистем в рамках стандартного подхода - выражения (5) и рассмотрения величин барьеров $U$, рассчитанных из измерений релаксации остаточной намагниченности. Для упрощения будем считать, подобно авторам работ [31,32], что $d_{\mathrm{FM}} \approx D$ (при $\left.D \approx 4 \mathrm{~nm}\right)$. Тогда при типичном для ферригидрита значении $M_{\mathrm{S}} \approx 20 \mathrm{Gs}[12,13,44]$ и $H_{\mathrm{EB}} \approx 1.25 \mathrm{kOe}$ для исследуемого образца FH-4 nm, из выражения (5) получим
$\sigma_{\mathrm{S}} \approx 0.01 \mathrm{erg} / \mathrm{cm}^{2}$. Если сопоставить величину потенциального барьера для условий ZFC $-U_{\mathrm{ZFC}}$ с подобной величиной в случае $\mathrm{FC}-U_{\mathrm{FC}}$, то их разница должна быть пропорциональной энергии обменной связи магнитных подсистем $E_{\mathrm{EB}}$

$$
E_{\mathrm{EB}} \sim U_{\mathrm{FC}}-U_{\mathrm{ZFC}}
$$

Величина $\left(U_{\mathrm{FC}}-U_{\mathrm{ZFC}}\right)$ из анализа данных по релаксации намагниченности составляет $\approx 150 \mathrm{~K}$. Видимо, значение $U_{\mathrm{ZFC}}$ несколько возрастет, если в условиях ZFC значение $H_{\max }$ будет большим $\left(\sim 10^{5} \mathrm{Oe}\right)$, и величина $M_{\mathrm{R} \_Z F C}$ будет близка к значению $M_{\mathrm{R} \_\mathrm{INF}}$ (см. рис. 4). Положим $\left(U_{\mathrm{FC}}-U_{\mathrm{ZFC}}\right) \sim 10^{2} \mathrm{~K}$, а площадь поверхности раздела $\mathrm{S}$ магнитных подсистем в АФМ-наночастице примем равной площади поверхности частицы $\mathrm{S} \sim \pi D^{2}$. Тогда из очевидного соотношения

$$
\sigma_{\mathrm{S}}=E_{\mathrm{EB}} / S
$$

получаем $\sigma_{\mathrm{S}} \approx 0.027 \mathrm{erg} / \mathrm{cm}^{2}$. Это значение примерно в два с половиной раза больше величины $\sigma_{\mathrm{S}}$, полученной в рамках стандартного подхода $\left(\sigma_{\mathrm{S}} \approx 0.01 \mathrm{erg} / \mathrm{cm}^{2}\right.$ из выражения (5)). Таким образом, наблюдается только качественное согласие, но оба способа страдают одним недостатком: неоднозначность в определении основных параметров (величин $d_{\mathrm{FM}}$ и $S$ ). В то же время, качественное согласие проведенных оценок $\sigma_{\mathrm{S}}$ позволяет говорить о возможности применения релаксационных процессов остаточной намагниченности для расчета энергии обменной связи магнитных подсистем в АФМ-наночастицах. Отметим, что полученное значение $\sigma_{\mathrm{S}}\left(0.01-0.03 \mathrm{erg} / \mathrm{cm}^{2}\right)$ близко по величине удельной энергии обменной связи наночастиц $\mathrm{NiO}$ $\left(0.03 \mathrm{erg} / \mathrm{cm}^{2}\right)$ [31] и превосходит таковую для наночастиц $\mathrm{CuO}\left(6 \cdot 10^{-5} \mathrm{erg} / \mathrm{cm}^{2}\right)$ [32].

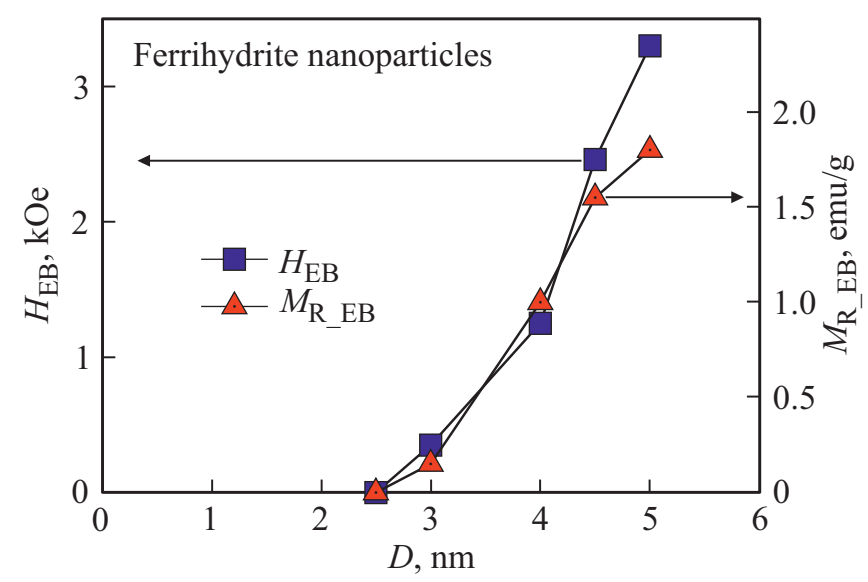

Рис. 6. Зависимости поля обменного смещения $H_{\mathrm{EB}}$ и „избыточной“ остаточной намагниченности $M_{\text {R_Eв }}$ (выражение (6)) от размера частиц ферригидрита $D$ по результатам работ $[42,43]$. Данные для образца, исследованного в этой работе, соответствуют $D=4 \mathrm{~nm}$. 
Итак, применение величин энергетических барьеров, определяемых из релаксации остаточной намагниченности, для выявления эффекта обменного смещения в АФМ-наночастицах является альтернативой обычному способу, в котором анализируется только поле обменного смещения. Отметим также возможность анализа „избыточной“ (выражение (6)). Для АФМ-наночастиц этот параметр является более значимым, чем для ФМ/АФМ-структур. Основанием этому утверждению служит схожесть зависимостей $H_{\mathrm{EB}}$ и $M_{\mathrm{R} \_\mathrm{B}}$ от размера частиц $D$ ферригидрита, представленная на рис. 6. На этом рисунке сведены данные, полученные в работах [42,43] на образцах биогенного и химического ферригидрита. Из данных рис. 6 следует симбатность указанных кривых, а именно: 1) рост $H_{\mathrm{EB}}$ и $M_{\mathrm{R} \_ \text {Ев }}$ с увеличением размера частиц и 2) пропорциональность величин $H_{\mathrm{EB}}$ и $M_{\mathrm{R} \_\mathrm{EB}}$ при одинаковом размере частиц. Отметим, что в работах [31,32] были получены зависимости $H_{\mathrm{EB}}$ от размеpa $D$ наночастиц $\mathrm{NiO}$ и $\mathrm{CuO}$. Эти зависимости также характеризуются ростом $H_{\mathrm{EB}}$ до некоторого значения $D$, а затем величина $H_{\mathrm{EB}}$ уменьшается (для субмикронных АФМ-частиц заметного эффекта обменного смещения не будет). Получение информации о поведении зависимостей $H_{\mathrm{EB}}(D), M_{\mathrm{R} \_ \text {ЕВ }}(D)$, о взаимосвязи $H_{\mathrm{EB}}(D)$ и $M_{\mathrm{R} \_ \text {ЕВ }}(D)$, о релаксации $M_{\mathrm{R} \_ \text {ЕВ }}(D)$ в дальнейшем дает возможность понимания механизмов эффекта обменного смещения в АФМ-наночастицах.

\section{4. Заключение}

АФМ-наночастицы характеризуются эффектом смещения петли магнитного гистерезиса после охлаждения во внешнем поле, начиная только с некоторого размера частиц, который для ферригидрита составляет $\sim 3 \mathrm{~nm}$. Указанный эффект связан с наличием обменной связи магнитных подсистем, формирующихся в антиферромагнитно упорядоченных наноразмерных частицах. Важной особенностью таких объектов является то, что эффект обменного смещения здесь приводит к сдвигу петли магнитного гистерезиса как по оси абсцисс, так и по оси ординат. Последнее обусловливает возможность анализа поведения остаточной намагниченности АФМ-систем в условиях обменного смещения. Для образцов наночастиц ферригидрита с различным средним размером „избыточная“ остаточная намагниченность - величина, аналогичная полю обменного смещения $H_{\mathrm{EB}}$, ведет себя от размера частиц $D$ схожим образом, как и $H_{\mathrm{EB}}(D)$.

Исследование релаксации остаточной намагниченности ферригидрита со средним размером наночастиц $4 \mathrm{~nm}$, проведенное в настоящей работе, позволило установить следующие факты. 1) Величина остаточной намагниченности заметно уменьшается с течением времени, как после охлаждения в поле, так и в условиях охлаждения без поля. 2) Относительное уменьшение остаточной намагниченности значительно больше в условиях охлаждения без поля. 3) Наблюдается логарифмическая зависимость релаксации остаточной намагниченности, начиная с $\sim 10^{2} \mathrm{~s}$. Последнее позволяет интерпретировать данные релаксации, используя общепринятое выражение (7), и получить значения энергии барьеров при наличии обменной связи (в условиях охлаждения в поле) и в обычных условиях (охлаждение без поля). Это дает возможность оценить величину энергии обменной связи магнитных подсистем (выражение (9)), которая по порядку величины согласуется с аналогичным значением, получаемым в ходе стандартного анализа величины поля обменного смещения (выражение (5)).

Таким образом, в настоящей работе на примере наночастиц ферригидрита продемонстрировано, что анализ остаточной намагниченности и ее релаксации в условиях эффекта обменного смещения дает возможность оценки величины энергии обменной связи магнитных подсистем, формирующихся в АФМ-наночастицах. Данный метод является альтернативой стандартному подходу, в котором анализируется только поле обменного смещения, определяемого из смещения петли гистерезиса $M(H)$ по оси полей.

\section{Конфликт интересов}

Авторы заявляют, что у них нет конфликта интересов.

\section{Список литературы}

[1] L. Néel, C.R. Acad. Sci. Paris 252, 4075 (1961).

[2] S. Mørup, D.E. Madsen, C. Fradsen, C.R.H. Bahl, M.F. Hansen. J. Phys.: Condens. Matter. 19, 213202 (2007).

[3] Ю.Л. Райхер, В.И. Степанов. ЖЭТФ 134, 514 (2008).

[4] N.J.O. Silva, V.S. Amaral, L.D. Carlos. Phys. Rev. B 71, 184408 (2005).

[5] M.S. Seehra, A. Punnoose. Phys. Rev. B 64, 132410 (2001).

[6] C. Rani, S.D. Tiwari. J. Magn. Magn. Mater. 385, 272 (2015).

[7] J.G.E. Harris, J.E. Grimaldi, D.D. Awschalom, A. Cholero, D. Loss. Phys. Rev. B 60, 3453 (1999).

[8] C. Gilles, P. Bonville, H. Rakoto, J.M. Broto, K.K.W. Wong, S. Mann. J. Magn. Magn. Mater. 241, 430 (2002).

[9] A.A. Lepeshev, I.V. Karpov, A.V. Ushakov, D.A. Balaev, A.A. Krasikov, A.A. Dubrovskiy, D.A. Velikanov, M.I. Petrov. J. Supercond. Nov. Magn. 30, 931 (2017).

[10] Д.А. Балаев, А.А. Дубровский, А.А. Красиков, С.И. Попков, А.Д. Балаев, К.А. Шайхутдинов, В.Л. Кириллов, О.Н. Мартьянов. ФТТ 59, 8, 1524 (2017).

[11] Д.А. Балаев, А.А. Дубровский, А.А. Красиков, С.В. Столяр, Р.С. Исхаков, В.П. Ладыгина, Е.Д. Хилажева. Письма ЖЭТФ 98, 3, 160 (2013).

[12] Д.А. Балаев, А.А. Красиков, А.А. Дубровский, С.В. Семёнов, О.А. Баюков, С.В. Столяр, Р.С. Исхаков, В.П. Ладыгина, Л.А. Ищенко. ЖЭТФ 146, 546 (2014).

[13] D.A. Balaev, A.A. Krasikov, A.A. Dubrovskiy, S.I. Popkov, S.V. Stolyar, O.A. Bayukov, R.S. Iskhakov, V.P. Ladygina, R.N. Yaroslavtsev. J. Magn. Magn. Mater. 410, 71 (2016).

[14] S.I. Popkov, A.A. Krasikov, D.A. Velikanov, V.L. Kirillov, O.N. Martyanov, D.A. Balaev. J. Magn. Magn. Mater. 483, 21 (2019). 
[15] S.I. Popkov, A.A. Krasikov, A.A. Dubrovskiy, M.N. Volochaev, V.L. Kirillov, O.N. Martyanov, D.A. Balaev. J. Appl. Phys. 126, 103904 (2019).

[16] C. Diaz-Guerra, M. Vila. J. Piqueras. Appl. Phys. Lett. 96 193105 (2010)

[17] Д.А. Балаев, А.А. Красиков, Д.А. Великанов, С.И. Попков, Н.В. Дубынин, С.В. Столяр, В.П. Ладыгина, Р.Н. Ярославцев. ФТТ 60, 1939 (2018).

[18] R.H. Kodama, A.E. Berkowitz. Phys. Rev. B 59, 6321 (1999).

[19] Yu.A. Koksharov, S.P. Gubin, I.D. Kosobudsky, G.Yu. Yurkov, D.A. Pankratov, L.A. Ponomarenko, M.G. Mikheev, M. Beltran, Y. Khodorkovsky, A.M. Tishin. Phys. Rev. B 63, 012407 (2000).

[20] E. Winkler, R.D. Zysler, M. Vasquez Mansilla, D. Fiorani. Phys. Rev. B 72, 132409 (2005).

[21] M. Tadic, D. Nikolic, M. Panjan, G.R. Blake. J. Alloys Comp. 647, 1061 (2015).

[22] С.В. Столяр, Д.А. Балаев, В.П. Ладыгина, А.И. Панкрац, Р.Н. Ярославцев, Д.А. Великанов, Р.С. Исхаков. Письма ЖЭТФ 111, 3, 197 (2020).

[23] M. Tadić, M. Panjan, D. Marković, I. Milošević, V. Spasojević. J. Alloys Comp. 509, 7134 (2011).

[24] А.С. Камзин, А.А. Валиуллин, В.Г. Семенов, Н. Das, N. Wakiy. ФTT, 61, 6, 1175 (2019).

[25] N.J.O. Silva, A. Millan, F. Palacio, E. Kampert, U. Zeitler, V.S. Amaral. Phys. Rev. B 79, 104405 (2009).

[26] W.P. Meiklejohn, C.P. Bean. Phys. Rev. 102, 1413 (1956).

[27] J. Nogués, I.K. Schuller. JMMM 192, 203 (1999).

[28] S. Giri, M. Patra, S. Majumdar. J. Phys.: Condens. Matter 23, 073201 (2011).

[29] J. Nogués, J. Sort, V. Langlais, V. Skumryev, S. Suriñach, J.S. Muñoz, M.D. Baró. Phys. Rep. 422, 65 (2005).

[30] S.A. Makhlouf, F.T. Parker, F.E. Spada, A.E. Berkowitz. J. Appl. Phys. 81, 8, 5561 (1997).

[31] S.A. Makhlouf, H. Al-Attar, R.H. Kodama. Solid State Commun. 145, 1 (2008).

[32] M.S. Seehra, A. Punnoose. Solid State Commun. 128, 299 (2003).

[33] A. Punnoose, M.S. Seehra. J. Appl. Phys. 91, 10, 7766 (2002).

[34] A. Punnoose, H. Magnone, M.S. Seehra, J. Bonevich. Phys. Rev. B 64, 174420 (2001).

[35] A.E. Bianchi, S.J. Stewart, R.D. Zysler, G. Punte. J. Appl. Phys. 112, 083904 (2012).

[36] S.A. Makhlouf, F.T. Parker, A.E. Berkowitz. Phys. Rev. B 55, R14717 (1997).

[37] A. Punnoose, T. Phanthavady, M.S. Seehra, N. Shah, G.P. Huffman. Phys. Rev. B 69, 054425 (2004).

[38] T.S. Berquó, J.J. Erbs, A. Lindquist, R.L. Penn, S.K. Banerjee. J. Phys.: Condens. Matter 21, 176005 (2009).

[39] M.S. Seehra, V. Singh, X. Song, S. Bali, E.M. Eyring. J. Phys. Chem. Solids 71, 1362 (2010).

[40] Д.А. Балаев, А.А. Красиков, А.А. Дубровский, С.В. Семёнов, С.И. Попков, С.В. Столяр, Р.С. Исхаков, В.П. Ладыгина, Р.Н. Ярославцев. ФТТ 58, 2, 280 (2016).

[41] N.J.O. Silva, V.S. Amaral, A. Urtizberea, R. Bustamante, A. Millán, F. Palacio, E. Kampert, U. Zeitler, S. de Brion, O. Iglesias, A. Labarta. Phys. Rev. B 84, 104427 (2011).

[42] D.A. Balaev, A.A. Krasikov, A.A. Dubrovskiy, S.I. Popkov, S.V. Stolyar, R.S. Iskhakov, V.P. Ladygina, R.N. Yaroslavtsev. J. Appl. Phys. 120, 183903 (2016).

[43] S.V. Stolyar, D.A. Balaev, V.P. Ladygina, A.A. Dubrovskiy, A.A. Krasikov, S.I. Popkov, O.A. Bayukov, Yu.V. Knyazev,
R.N. Yaroslavtsev, M.N. Volochaev, R.S. Iskhakov, K.G. Dobretsov, E.V. Morozov, O.V. Falaleev, E.V. Inzhevatkin, O.A. Kolenchukova, I.A. Chizhova. J. Supercond. Nov. Magn. 31, 2297 (2018).

[44] С.В. Столяр, Р.Н. Ярославцев, Р.С. Исхаков, О.А. Баюков, Д.А. Балаев, А.А. Дубровский, А.А. Красиков, В.П. Ладыгина, А.М. Воротынов, М.Н. Волочаев. ФТТ 59, 3, 538 (2017).

[45] Ю.Л. Райхер, В.И. Степанов, С.В. Столяр, В.П. Ладыгина, Д.А. Балаев, Л.А. Ищенко, М. Балашою. ФТТ 52, 277 (2010).

[46] С.В. Столяр, О.А. Баюков, В.П. Ладыгина, Р.С. Исхаков, Л.А. Ищенко, В.Ю. Яковчук, К.Г. Добрецов, А.И. Поздняков, О.Е. Пиксина. ФТТ 53, 1, 97 (2011).

[47] L. Anghel, M. Balasoiu, L.A. Ishchenko, S.V. Stolyar, T.S. Kurkin, A.V. Rogachev, A.I. Kuklin, Y.S. Kovalev, Y.L. Raikher, R.S. Iskhakov, G. Duca. J. Phys. Conf. Ser. 351, 12005 (2012).

[48] Д.А. Балаев, А.А. Красиков, С.В. Столяр, Р.С. Исхаков, В.П. Ладыгина, Р.Н. Ярославцев, О.А. Баюков, А.М. Воротынов, М.Н. Волочаев, А.А. Дубровский. ФТТ 58, 9, 1724 (2016).

[49] А.Д. Балаев, Ю.В. Бояршинов, М.М. Карпенко, Б.П. Хрусталев. ПТЭ 3, 167 (1985).

[50] A.P. Malozemoff. J. Appl. Phys. 63, 3874 (1988).

[51] J. Tejada, X.X. Zhang, E.M. Chudnovsky. Phys. Rev. B 47, 14977 (1993).

[52] R. Prozorov, Y. Yeshurun, T. Prozorov, A. Gedanken. Phys. Rev. B 59, 6956 (1999).

[53] J. Tejada, X.X. Zhang. J. Phys.: Condens. Matter. 6, 263 (1994).

Редактор Ю.Э. Китаев 